\begin{tabular}{|c|c|c|c|c|}
\hline$a_{11}$ & $\begin{array}{l}a_{12} \\
a_{22}\end{array}$ & $\begin{array}{ccc}a_{13} & \cdots & a_{1 n} \\
a_{23} & \cdots & a_{2 n} \\
a_{38} & \cdots & a_{3 n} \\
& \cdot \\
& \cdot \\
& & \cdot \\
& & a_{n n}\end{array}$ & $\begin{array}{l}g_{1} \\
g_{2} \\
g_{8} \\
\dot{.} \\
\dot{.} \\
g_{n}\end{array}$ & $\begin{array}{l}\overline{\bar{g}} \\
\overline{g_{2}} \\
\overline{g_{3}} \\
\cdot \\
\cdot \\
\overline{g_{n}}\end{array}$ \\
\hline$s_{11}$ & $\begin{array}{l}s_{12} \\
s_{22}\end{array}$ & 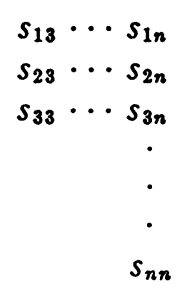 & $\begin{array}{c}k_{1} \\
k_{2} \\
k_{3} \\
\cdot \\
\dot{\cdot} \\
\dot{\cdot} \\
k_{n}\end{array}$ & $\begin{array}{c}\overline{k_{1}} \\
\overline{k_{2}} \\
k_{3} \\
\cdot \\
\dot{\cdot} \\
\dot{\bar{k}}\end{array}$ \\
\hline$\frac{x_{1}}{x_{1}}$ & $\frac{x_{2}}{x_{2}}$ & $\frac{x_{3} \cdots x_{n}}{\bar{x}_{3} \cdots \overline{x_{n}}}$ & & \\
\hline
\end{tabular}

It should be noted that the diagonal elements of the $S$ matrix involve square roots which may lead to imaginary elements. This would never occur with normal equations but could arise when $A$ is an arbitrary symmetric matrix. However, the occurrence of pure imaginary elements in $S$ causes no difficulty in applying the method.

JACK LADERMAN

NBSCL

1 Tadeusz Banachiewicz, (a) "Principes d'une nouvelle technique de la méthode des moindres carrés"; (b) "Méthode de résolution numérique des équations linéaires, du calcul des déterminants et des inverses, et de réduction des formes quadratiques," Akademija Umiejetnosci, Krakow, Wydzial Matematyczno-przyrodniczy, Bull. Intern., s.A., Sci. Math., 1938 , p. $134-135 ; 393-404$.

${ }^{2}$ P. S. DWYER, "A matrix presentation of least squares and correlation theory with matrix justification of improved methods of solution," Annals Math. Stat., v. 15, 1944, p. 82-89; and "The square root method and its use in correlation and regression," Amer. Stat. Assn., Jn., v. 40, 1945, p. 493-503.

${ }^{3}$ David B. Duncan and John F. Kenney, On the Solution of Normal Equations and Related Topics. Ann Arbor, Edwards Bros., 1946, 35 p.

\title{
Coefficients for Expressing the First Twenty- Four Powers in Terms of the Legendre Polynomials
}

The following table of coefficients gives the exact expression for $x^{n}$, $n=0(1) 24$, in terms of Legendre polynomials $P_{m}(x)$ (written simply as $P_{m}$ ). This extends the previous short tables (inadequate for many needs) which are given in T. M. MAcRoBert, Spherical Harmonics, London, 1927, p. 96, $n=0(1) 10$; in W. E. BYERLY, Elementary Treatise on Fourier's Series and Spherical, Cylindrical and Ellipsoidal Harmonics. Boston, 1895, p. 179, $n=0(1) 8$; and in E. W. HoBson, Theory of Spherical and Ellipsoidal 
Harmonics. Cambridge, 1931, p. $45, n=0(1) 7$. The coefficients were calculated from the following formulae:

For $n$ even:

$$
\begin{aligned}
x^{n}=\frac{1}{(n+1)} P_{0}+5 & \frac{n}{(n+1)(n+3)} P_{2}+9 \frac{n(n-2)}{(n+1)(n+3)(n+5)} P_{4} \\
& +\cdots+(2 n+1) \frac{n(n-2) \cdots 2}{(n+1)(n+3) \cdots(2 n+1)} P_{n} .
\end{aligned}
$$

For $n$ odd:

$$
\begin{aligned}
x^{n}=\frac{3}{(n+2)} P_{1}+7 & \frac{(n-1)}{(n+2)(n+4)} P_{3}+11 \frac{(n-1)(n-3)}{(n+2)(n+4)(n+6)} P_{5} \\
& +\cdots+(2 n+1) \frac{(n-1)(n-3) \cdots 2}{(n+2)(n+4) \cdots(2 n+1)} P_{n} .
\end{aligned}
$$

As a check, all the coefficients of $P_{m}$ (except those of $P_{0}$ or $P_{1}$ ) were recalculated by a different method from the expression

$$
(2 m+1) \frac{n(n-1) \cdots(n-m+2)}{(n+m+1)(n+m-1) \cdots(n-m+3)} .
$$

An additional overall check, for each $n$, was provided by taking the sum of the coefficients of $P_{m}$, which is equal to 1 .

These coefficients are useful in obtaining an approximation for polynomials of high degree, which is best in a well-known least square sense. When any function in the interval $[a, b]$ (which is first transformed into $[-1,1])$ is expanded in terms of Legendre polynomials, (denoting the normalized function by $f(x)$ and the partial sum of degree $r$ by $q_{r}(x)$ ), then

$$
\int_{-1}^{1}\left|f(x)-q_{r}(x)\right|^{2} d x<\int_{-1}^{1}\left|f(x)-r_{r}(x)\right|^{2} d x,
$$

where $r_{r}(x)$ is any polynomial of degree $\leqslant r$ that differs from $q_{r}(x)$. For methods of using these coefficients, one might consult the very instructive article by C. LANCzos, "Trigonometric interpolation of empirical and analytic functions," J. Math. Physics, v. 17, 1938, p. 123-199. Lanczos deals mainly with "economization" using Chebyshev polynomials, but indicates (p. 143-145) that his same methods are applicable to Legendre polynomials, if one desires approximation in this least square sense.

$$
\begin{aligned}
1 & =P_{0} \\
x & =P_{1} \\
3 x^{2} & =P_{0}+2 P_{2} \\
5 x^{3} & =3 P_{1}+2 P_{3} \\
35 x^{4} & =7 P_{0}+20 P_{2}+8 P_{4} \\
63 x^{5} & =27 P_{1}+28 P_{8}+8 P_{5} \\
231 x^{6} & =33 P_{0}+110 P_{2}+72 P_{4}+16 P_{6} \\
429 x^{7} & =143 P_{1}+182 P_{3}+88 P_{6}+16 P_{7} \\
6435 x^{8} & =715 P_{0}+2600 P_{2}+2160 P_{4}+832 P_{6}+128 P_{8} \\
12155 x^{9} & =3315 P_{1}+4760 P_{8}+2992 P_{5}+960 P_{7}+128 P_{9} \\
46189 x^{10} & =4199 P_{0}+16150 P_{2}+15504 P_{4}+7904 P_{6}+2176 P_{8}+256 P_{10} \\
88179 x^{11} & =20349 P_{1}+31654 P_{3}+23408 P_{5}+10080 P_{7}+2432 P_{9}+256 P_{11}
\end{aligned}
$$




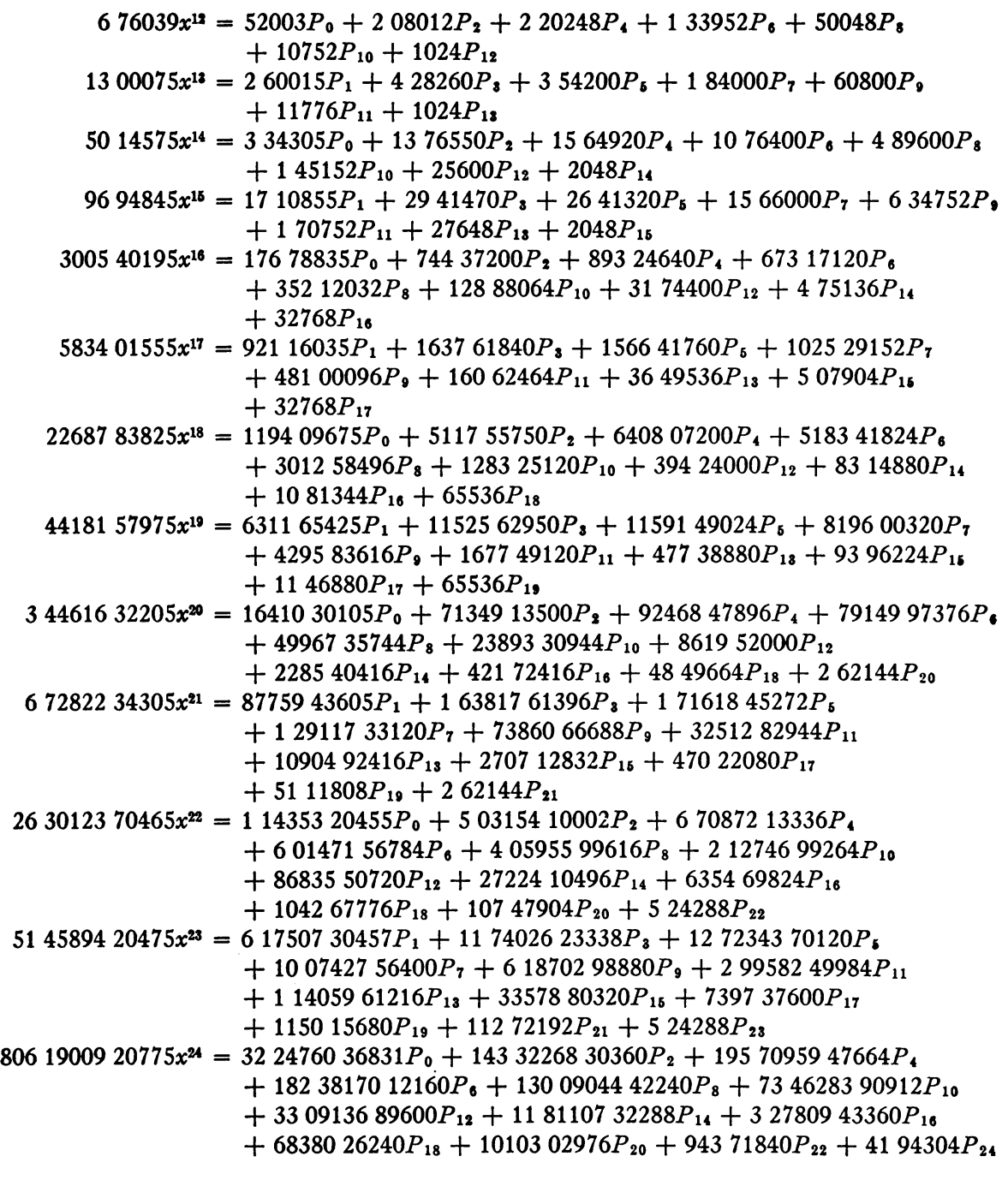

NBSCL

Herbert E. Salzer

\title{
A New Approximation to $\pi$
}

\author{
(conclusion)
}

In $M T A C$, v. 2, p. 320 , it was announced that the values of $\pi$ and $\tan ^{-1} \frac{1}{5}$, in our previous joint article with Mr. Smith, p. 245-248, called for correction beyond 722D. We are now in a position to guarantee the accuracy of our $808 \mathrm{D}$ values of $\pi, \tan ^{-1} \frac{1}{5}, \tan ^{-1} \frac{1}{238}, \tan ^{-1} \frac{1}{4}, \tan ^{-1} \frac{1}{20}$, and $\tan ^{-1} \frac{1}{1885}$.

This certainty was achieved by $\mathrm{Mr}$. Ferguson carrying through his calculations to $812 \mathrm{D}$, with the independent formula which he had been using. In this way he discovered certain errors in the work of Dr. Wrench. 